\title{
Comment transformer un référentiel de littératie numérique en un outil de médiation pédagogique? Analyse pratique.
}

\author{
Auteurs \\ Hervé Platteaux, Centre NTE DIT - Université de Fribourg - Suisse, \\ herve.platteaux@unifr.ch \\ Laurent Moccozet, Centre Universitaire d'Informatique - Université de \\ Genève - Suisse, \\ laurent.moccozet@unige.ch \\ Arik Levy, Université de Genève - Suisse, \\ arik.levy@unige.ch \\ Laura Molteni, Centre NTE DIT - Université de Fribourg - Suisse, \\ laura.molteni@unifr.ch \\ Giulia Ortoleva, TECFA - Université de Genève - Suisse, \\ giulia.ortoleva@unige.ch \\ Patrick Roth, Pôle e-learning - Université de Genève - Suisse, \\ patrick.roth@unige.ch
}

Emmanuelle Salietti, Centre NTE DIT - Université de Fribourg - Suisse, emmanuelle.salietti@unifr.ch

Elsa Sancey, Pôle e-learning - Université de Genève - Suisse, elsa.sancey@unige.ch 


\section{REVUE HYBRIDE DE L'ÉDUCATION}

\section{Résumé}

Les référentiels de littératie numérique sont des outils de médiation. Ceux issus de la recherche ou d'instances éducatives précisent des dimensions («pensée critique », «production de contenus numériques ») facilitant une médiation stratégique. Comment transformer ces référentiels pour faciliter une médiation pédagogique? Deux éléments apparaissent essentiels : 1) décrire les compétences en composantes hiérarchiques et 2) contextualiser et approfondir leurs descriptions. L'article témoigne d'actions d'innovation menées en parallèle dans les Universités de Fribourg et de Genève pour produire des ressources sur les compétences numériques à partir de référentiels pédagogiques à l'intention des étudiant·e.s de baccalauréat et de maîtrise.

Mots-clés: référentiel de compétences; compétence numérique; médiation pédagogique ; étudiant·e.s universitaires ; outil pédagogique 


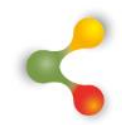

\section{REVUE HYBRIDE DE L'ÉDUCATION}

\section{Médiation des compétences numériques \\ 1.1 Problématique}

Le travail présenté dans cet article décrit une méthode pour créer un outil de médiation pédagogique à partir d'un référentiel générique de littératie numérique. Notons que nous considérons seulement le contexte du développement des compétences numériques des étudiant·e.s universitaires.

Notre constat de départ est que les référentiels de compétences venant de la recherche (Carretero et al., 2017 ; Van Laar et al., 2017 ; Voogt et Roblin, 2012) ou d'instances éducatives (Gouvernement du Québec, 2019 ; Gouvernement de l'Ontario, 2016) sont trop génériques pour servir d'outil de médiation pédagogique, mais ils précisent des dimensions (par exemple: "pensée critique», "production de contenus numériques ») fixant une référence pour une médiation stratégique (orientation, guidage). Ils sont donc nécessaires pour concevoir des formations ou des ressources d'apprentissage visant le développement des compétences numériques.

Une méthode transformant des référentiels génériques en outil de médiation pédagogique serait alors fort utile. Celle-ci n'est pas simple, car les référentiels de compétences sont composés de nombreux éléments : "des listes de capacités, connaissances, savoir-faire, savoir-être, habiletés, aptitudes, qualités, etc., et compétences, plus ou moins généraux, décontextualisés et non hiérarchisés. " (Mayen et al., 2010, p. 32-33).

\subsection{Contexte de l'innovation menée Contexte de l'apprentissage des compétences numériques}

Les référentiels de compétences numériques ont un rôle particulier dans l'enseignement supérieur, car l'apprentissage de celles-ci y reste largement «informel». D'une part, le «domaine » des compétences numériques ne s'y décline pas en diplômes et très peu en cours/enseignements, contrairement aux autres domaines dotés de référentiels. D'autre part, considérer les étudiant.e.s comme des digital natives (Jones, 2013) a fait penser qu'il n'y avait pas besoin d'apprentissage « formel » dans ce domaine.

Aujourd'hui, ce domaine s'intègre notamment dans certains ateliers méthodologiques inscrits dans les cursus. Des programmes nationaux se mettent aussi en place pour développer les compétences numériques. De plus, l'idée des natives évolue : «The overall conclusion is that digital natives are not necessarily information literate, and that IL should be promoted with hands-on and minds-on courses based on IL standards » (Sorgo et al., 2017). 


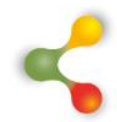

\section{REVUE HYBRIDE DE L'ÉDUCATION}

Toutefois, le développement et l'utilisation de ces compétences résultent encore le plus souvent d'activités faites en autonomie par les étudiant.e.s alors que les institutions ont un rôle important à y jouer (Littlejohn et al., 2013), en particulier rendre accessibles des ressources d'aide à l'apprentissage comme les référentiels.

L'aspect transversal des compétences numériques implique qu'elles ont besoin d'être répertoriées de façon interdisciplinaire, car les compétences numériques apprises pas les étudiant·e.s dans un domaine vont pouvoir être mobilisées dans un autre.

Ces changements en cours et ce besoin de répertorier les compétences numériques donnent des rôles particulièrement importants aux référentiels dans ce domaine.

\section{Quels rôles peuvent jouer les référentiels de compétences?}

Les référentiels de littératie numérique qui proviennent d'un ministère ou de la littérature servent, le plus souvent, d'outils de médiation stratégique pour guider et orienter, par exemple, la définition des programmes de cours et de formations.

Dans ces référentiels, la description des compétences est générique, c'est-à-dire courte et non dédiée à un contexte d'enseignement particulier, comme dans le DigComp2.1: "Browsing, searching and filtering data, information and digital content. To articulate information needs, to search for data, information and content in digital environments, to access and navigate between them. To create and update personal search strategies. » (Carretero et al., 2017, p. 23) Ayant ce rôle de médiation stratégique, ces référentiels génériques doivent constituer une base pour élaborer des cours et des formations mettant en œuvre ces programmes et devenir ainsi des outils facilitant une médiation pédagogique.

Dans cette optique, notre attention est retenue par une analyse montrant que des référentiels courts ont une utilité de communication (Vandeweerd et al., 2014). Pour élaborer un dispositif de formation sur les compétences numériques, les référentiels courts facilitent la communication entre les expert.e.s de l'enseignement d'un domaine disciplinaire et du domaine numérique. D'autre part, Vandeweerd et ses collaborateurs (2014) voient que l'utilisation des référentiels génériques pour d'autres aspects pédagogiques que la communication semble empêchée parce que leurs descriptions des compétences sont trop générales et pas assez précises.

À l'encontre, la logique de regroupement en domaines des compétences numériques proposée par un référentiel générique est à exploiter du point de vue pédagogique. Ces domaines sont, par exemple, 


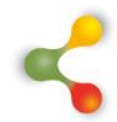

\section{REVUE HYBRIDE DE L'ÉDUCATION}

la recherche d'informations numériques ou la création de documents numériques. Cette logique esquisse l'alignement pédagogique à créer (entre objectifs, activités, évaluation et accompagnement) dans des dispositifs d'apprentissage pour "qu'étudiant·e.s et enseignant.e.s construisent une vision partagée du dispositif de formation et de ses objectifs. » (Peraya et al., 2014, p. 24). Aussi, en détaillant les descriptions de compétences proposées par un référentiel générique, on peut faciliter "la clarification du contrat didactique» ou la "systématique dans l'évaluation des compétences » (Postiaux et al., 2010, p. 23).

Ainsi, les référentiels de compétences numériques pourront jouer des rôles dans la médiation pédagogique se développant dans les équipes élaborant un dispositif de formation, et entre les enseignant.e.s et les étudiant·e.s utilisant celui-ci.

\subsection{Deux grandes notions théoriques}

D'abord, pour définir ces rôles et les «opérationnaliser », il faut approfondir la notion de médiation pédagogique ainsi que les méthodes de construction possibles d'un référentiel de compétences permettant de passer d'un référentiel générique à un référentiel plus détaillé. C'est le but de la présente section de notre article.

\section{Médiation pédagogique, quoi et comment?}

Analyser comment un référentiel de compétences peut agir comme un outil de médiation pédagogique place l'apprentissage et l'apprenant·e au cœur de notre travail, car «le processus d'apprentissage s'établit entre le sujet et l'objet par l'entremise d'un système objectif de régulation (la médiation) fondé sur la parole en tant que discours et sur l'action humaine en tant que processus de production sociale » (Lenoir, 2009, p. 20). Nous sommes donc dans un cadre socioconstructiviste (Chini, 2001) où le langage réfère " $[\mathrm{au}]$ rapport social en situation - un rapport social d'objectivation - qui s'établit entre un.e enseignant.e et ses élèves à propos des objets d'apprentissage prévus" (Lenoir, 2009, p. 12). II médiatise tous les processus psychocognitifs intervenant dans un apprentissage (Vygotski, 1934). L'action, elle, est une des racines du processus d'apprentissage qui prend son point de départ: "dans un complexe indissociable d'interactions entre l'objet et le sujet, et qui est fourni par l'action » (Piaget, 1971, p. 201).

Le terme «médiation pédagogique » trouve alors son sens : «Un processus d'apprentissage, en tant que processus d'objectivation, n'est donc pas simplement appropriation, mais avant tout construction médiatisée d'un objet. » (Lenoir, 2009, p. 22). De plus, ce terme montre que "Pour décrire et comprendre la pratique d'enseignement comme une intervention éducative [...] il faut l'associer à plusieurs autres concepts, 


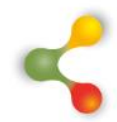

\section{REVUE HYBRIDE DE L'ÉDUCATION}

dont ceux de médiation cognitive, de situation, de dispositif instrumental, mais aussi procédural (facilitateurs et démarches). " (Lenoir, 2009, p. 18). Peraya et ses collègues (2014) placent la notion de dispositif au cœur de la médiation, car il médiatise toutes les interactions qu'a un sujet avec le monde pour apprendre. Rabardel et Samurcay (2001) montrent comment s'opérationnalise cette médiation : l'artefact devient instrument avec ses schèmes sociaux d'utilisation.

L'enseignant·e agit en intervenant, sur le plan d'une médiation psychopédagogique, pour devenir un constructeur de sens pour l'apprenant-e (Tardif et Lessard, 1999). Chez l'apprenant·e, l'activation d'une médiation cognitive est favorisée grâce au dispositif d'apprentissage et à une stratégie de médiation organisant les actions de l'intervenant "destinées à amener l'apprenant à résoudre un conflit, potentiel ou réel, d'apprentissage » (Myara, 2017, p. 153). Ainsi, la médiation pédagogique est régulatrice ; elle « doit mettre en relation l'apprenant et la matière, mais de manière encore plus importante, elle doit révéler l'apprenant à luimême. " (Chini, 2001, p. 137). Elle se situe ainsi dans le temps, car, comme le souligne Myara (2018a), l'intervention de l'enseignant e peut être faite avant l'action de l'apprenant·e (pour anticiper et prévoir), pendant (pour guider, autoévaluer) et après (pour évaluer ou autoévaluer). Dans cette régulation métacognitive, le langage doit surtout médiatiser le rapport au savoir de l'apprenant-e pour que celui-ci conceptualise ce savoir (Chini, 2001). Pour cela, Varinot (2012) préconise d'employer un mode interrogatif du langage pour conduire l'apprenant·e à «verbaliser ses procédures mentales " et l'enseignant·e à "superviser le processus d'évaluationrégulation suivi par l'élève et à maintenir celui-ci orienté vers le but à atteindre ", à « expliciter directement le but à atteindre » et à « guider l'élève vers la prise en considération des données de la tâche, de ses contraintes et de ses ressources » (p. 75).

\section{Compétences et référentiels de compétences}

Introduisons d'abord la notion de capacité : « Une capacité, c'est le pouvoir, l'aptitude à faire quelque chose. C'est une activité que l'on exerce. » (Rogiers et De Ketele, 2001, p. 50). Suite à cette définition, on peut introduire celle de la notion de compétence :

- «La compétence est la mobilisation ou l'activation de plusieurs savoirs, dans une situation et un contexte donnés 》 (Le Boterf, 1999);

- Une compétence est « un savoir-agir complexe prenant appui sur la mobilisation et la combinaison efficaces d'une variété de ressources internes et externes à l'intérieur d'une famille de situations » (Tardif, 2006, p. 22) ;

- Une compétence est « la possibilité, pour un individu, de mobiliser de manière intériorisée un ensemble intégré de ressources en vue 


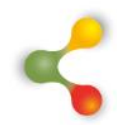

\section{REVUE HYBRIDE DE L'ÉDUCATION}

de résoudre une famille de situations-problèmes » (Scallon, 2004, p. 105).

Si une personne a une compétence parce qu'elle sait mettre en œuvre (mobiliser) une capacité dans une situation, la description d'une compétence est intrinsèquement liée au contexte associé à la situation dans laquelle une tâche doit être effectuée : «Une compétence se définit comme un système de connaissances, conceptuelles et procédurales, organisées en schémas opératoires et qui permettent, à l'intention d'une famille de situations, l'identification d'une tâche-problème et sa résolution par une action efficace. » (Gillet, 1991, p. 69).

Un référentiel présente une structure hiérarchique organisée « en domaines de compétences, en compétences et en indicateurs de compétences » (Carignan et al., 2014, p. 11). Or les référentiels génériques esquissent seulement cette structure. II faut détailler tous les contextes, du plus large au plus focalisé, des tâches et des situations du domaine de compétences pour compléter la hiérarchie en atteignant le niveau des indicateurs comme le montre un travail antérieur (Platteaux et al., 2019). On transforme ainsi un référentiel générique en outil de médiation pédagogique en complétant sa structure hiérarchique basée sur un alignement pédagogique. De plus, en pensant à un référentiel de compétences comme à une grille critériée, on précise les critères d'évaluation des compétences: "Rubrics make clear to students the expectations of their instructors and provide consistent and transparent performance criteria » (Lowe et al., 2015, p. 492).

Pour décrire toutes les composantes hiérarchiques d'une compétence, la difficulté à dépasser est qu' :

Une compétence est foncièrement hétérogène : elle peut mettre en jeu des connaissances déclaratives, des connaissances procédurales, des automatismes, des raisonnements, des données retenues en mémoire de travail, des schèmes sensorimoteurs ou n'importe quel assemblage de ces éléments ou tout ce qu'on voudra. (Rey, 2006, p. 95).

De plus, il s'agit de trouver l'équilibre entre généralité pour l'opérabilité (usability) et détail pour la fiabilité (reliability) (Rusman et Dirkx, 2017). Des règles systématiques sont donc nécessaires pour pouvoir écrire les compétences avec un niveau de détail suffisant, en mettant en évidence leurs niveaux de difficulté et la variété des aspects psychocognitifs liés aux différentes sous-compétences (celles des sous-contextes de la structure hiérarchique).

Si nous nous concentrons sur l'écriture des aspects de la compétence liés à la tâche de travail, nous adoptons les travaux de 


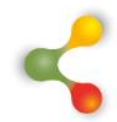

\section{REVUE HYBRIDE DE L'ÉDUCATION}

Paquette (2002) où les compétences sont décrites comme la mobilisation d'habiletés (quatre niveaux: Réception, Reproduction, Création, Autogestion) sur des connaissances (trois types: Concept, Procédure, Principe [plus les Faits]). Notons que les quatre niveaux d'habiletés peuvent être précisés en 10 classes au total : Réception (en deux classes : prêter attention, repérer ou mémoriser), Reproduction (en trois classes), Création (en trois) et Autogestion (en deux). Cette méthode concrétise l'idée de la centralité des actions humaines dans la médiation pédagogique et celle de leur évaluation, car les composantes des compétences (habiletés $x$ connaissances) sont écrites avec une taxonomie de verbes d'action (connaître, analyser, synthétiser, etc.). De plus,

il nous appert essentiel que le médiateur, étant déjà expert de son contenu, se dote d'un modèle et d'un cadre de référence qui représentent les mécanismes cognitifs afin de pouvoir expliquer les diverses actions mentales nécessaires à la réalisation d'une tâche. (Myara, 2018b, p. 82)

Pour écrire une composante d'une compétence, on choisit le niveau et la classe d'habileté et le type de connaissance correspondant à cette composante. On peut devoir appliquer plusieurs fois cette procédure pour pouvoir décrire tous les aspects d'une composante. Donnons comme exemples quelques aspects concrets d'une tâche de recherche d'information bibliographique. Un·e étudiant·e doit connaître l'existence de bases de données bibliographiques scientifiques (Réception x Concept). Elle ou il doit également savoir qu'il faut mener une recherche bibliographique avec de telles bases de données parce qu'elles sont systématiques et complètes (Réception x Principe) à la différence de Google qui a un autre usage. Un tout autre aspect est la maîtrise nécessaire de la création d'une requête faite avec des mots-clés reliés avec des «ET», des «OU », etc. (Reproduction x Procédure).

Si nous nous concentrons sur l'écriture des aspects de la compétence liée à la situation de travail, nous nous inspirons de la démarche proposée par Vandeweerd et al. (2014): «CFs should be constructed by associating knowledge, skills, and attitudes directly with the families of situations or by defining transverse competencies which can be exerted within the context of these families. " (p. 8). Nous nous appuyons également sur un cadre de référence simplifié de stratégies d'apprentissage élaboré par Bégin (2008) pour prendre en compte « des situations où l'apprenant doit traiter les informations dans le but de les apprendre ", mais aussi « des situations de performance, de production de connaissances ou d'exécution de tâches " (p. 58): stratégies métacognitives (anticiper, s'autoréguler), stratégies cognitives (de traitement: sélectionner, répéter, décomposer, comparer, élaborer, organiser; d'exécution : évaluer, vérifier, produire, traduire). Prenons ici l'exemple d'une situation d'apprentissage usuelle : un·e étudiant.e assiste 


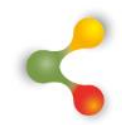

\section{REVUE HYBRIDE DE L'ÉDUCATION}

à un cours ex cathedra dans un amphithéâtre. Elle ou il devra être capable de sélectionner les éléments importants du discours de l'enseignant·e afin de produire des notes, de traduire les propos de l'enseignant $e$ avec ses mots et de comparer ultérieurement ses notes avec celles d'un e collègue pour vérifier leur exactitude et leur précision.

\section{Construction d'un référentiel de compétences numériques pédagogique}

En utilisant les bases théoriques qui précèdent, nous avons mené dans nos universités une innovation visant à construire un référentiel de compétences numériques pouvant être utilisé comme outil de médiation pédagogique pour élaborer et mettre en place de ressources aidant le développement des compétences numériques des étudiant.e.s. Cette section montre le déroulement des étapes de cette construction.

Cette approche métier peut se généraliser et décliner des référentiels spécialisés pour d'autres populations du monde académique : enseignant.e.s, chercheur.e.s, en contextualisant à chaque fois des situations de ces métiers, des domaines ou des facultés.

Référentiel de compétences dans les activités du métier de l'étudiant·e

À l'Université de Fribourg, la construction du référentiel de compétences numériques accompagnait la création d'un cours aidant les étudiant·e.s en baccalauréat et maîtrise à développer ces compétences. Elle s'est déroulée en trois étapes de travail (tableau 1) avec différentes actions concrètes effectuées sur les composantes du référentiel, structurée sur la description des tâches d'apprentissage des étudiant·e.s. On utilise ici la méthode d'écriture des compétences de Paquette (2002). 


\section{REVUE HYBRIDE DE L’ÉDUCATION}

\section{Tableau 1 : Construction d'un référentiel contextualisé par les activités de travail}

\begin{tabular}{|c|c|}
\hline \multicolumn{2}{|c|}{ Étape 1 : Focalisation et alignement des compétences } \\
\hline $\begin{array}{l}\text { Actions concrètes effectuées } \\
\text { - prendre le référentiel générique } \\
\text { - adapter le référentiel générique }\end{array}$ & $\begin{array}{l}\text { Éléments définis dans le référentiel } \\
\text { - intitulés des compétences } \\
\text { génériques descriptions courtes des } \\
\text { - compétences génériques } \\
\text { - hiérarchie des compétences } \\
\text { génériques en } \\
\text { - groupes de compétences } \\
\text { - compétences dans chaque } \\
\text { groupe }\end{array}$ \\
\hline \multicolumn{2}{|c|}{ Étape 2 : Contextualisation des compétences pour chaque activité } \\
\hline $\begin{array}{l}\text { Actions concrètes effectuées } \\
\text { - rendre le référentiel spécifique } \\
\text { - contextualiser avec les activités } \\
\text { - contextualiser avec les domaines } \\
\text { - contextualiser avec les temporalités }\end{array}$ & $\begin{array}{l}\text { Éléments définis dans le référentiel } \\
\text { - intitulés des compétences } \\
\text { spécifiques } \\
\text { - descriptions courtes des } \\
\text { compétences spécifiques } \\
\text { - hiérarchie des compétences } \\
\text { spécifiques contextualisées par les } \\
\text { activités } \\
\text { - groupes de compétences : } 1 \\
\text { compétence pour } 1 \text { activité } \\
\text { - sous-compétences de chaque } \\
\text { activité (pas systématique) }\end{array}$ \\
\hline \multicolumn{2}{|c|}{ Étape 3 : Approfondissement des activités pour chaque compétence } \\
\hline $\begin{array}{l}\text { Actions concrètes effectuées } \\
\text { - lister les sous-compétences de chaque } \\
\text { compétence } \\
\text { - décrire précisément chaque sous- } \\
\text { compétence } \\
\text { - en conséquence, décrire précisément } \\
\text { chaque compétence }\end{array}$ & $\begin{array}{l}\text { Éléments définis dans le référentiel } \\
\text { - descriptions approfondies des } \\
\text { compétences } \\
\text { - descriptions approfondies des } \\
\text { sous-compétences : } \\
\text { - définition des habiletés et } \\
\text { connaissances mobilisées } \\
\text { - définition des actions mentales } \\
\text { impliquées } \\
\text { - hiérarchie systématique des } \\
\text { compétences et sous-compétences }\end{array}$ \\
\hline
\end{tabular}




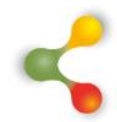

\section{REVUE HYBRIDE DE L'ÉDUCATION}

À la fin de l'étape 1, on obtient les compétences génériques avec leur alignement de base retiré directement des référentiels génériques utilisés à l'Université de Fribourg (Carretero et al., 2017 ; JISC team, 2014 ; Van Laar et al., 2017). Ces groupes de compétences forment la structure hiérarchique de base du référentiel.

Nous avons distingué les groupes suivants: Culture informatique, Littératie informationnelle (IL : trois compétences génériques), Création de contenus numériques (DCC: cinq compétences génériques), Communication et Collaboration ( $C C$ : six compétences génériques) et Résolution de problèmes (RP : six compétences génériques). Pour dénommer cette étape, nous employons les notions de focalisation et d'alignement pour indiquer qu'un groupe définit une certaine catégorie de compétences. Détaillons un exemple: Le groupe "Littératie Informationnelle » comporte les trois compétences génériques ${ }^{1}$ :

1. Naviguer, chercher et filtrer des données, informations et contenus numériques ;

2. Évaluer des données, informations et contenus numériques ;

3. Entreposer et Gérer des données, informations et contenus numériques.

À la fin de l'étape 2, on a les intitulés et les descriptions courtes des compétences mobilisées de chaque tâche-activité de travail réalisée régulièrement par les étudiant·e.s faisant un cursus à l'université. Autrement dit, on a contextualisé le référentiel générique au métier de l'étudiant·e. Pour le cours sur les compétences numériques de l'Université de Fribourg, à ce stade, on a pris chaque groupe de compétences génériques et défini les activités des étudiant-e.s faisant partie de ce groupe, c'est-à-dire les compétences spécifiques. Ce sont les objectifs d'apprentissage visés par le cours (IL : neuf activités ; DCC : 10 activités ; CC : 16 activités; RP : 13 activités). Continuons l'exemple du groupe "Littératie Informationnelle» pour la tâche "Recherche d'information bibliographique » du métier de l'étudiant·e. Les compétences contextualisées des trois compétences génériques sont :

1. Choisir et Utiliser des bases de données bibliographiques scientifiques pour faire une recherche d'information thématique ;

2. Évaluer des documents bibliographiques trouvés selon différents critères de qualité : validité, pertinence, etc. ;

3. Entreposer et gérer des références bibliographiques.

Le référentiel de compétences numériques construit est pensé pour toutes et tous les étudiantes et les étudiants à l'université. On peut aussi contextualiser plus précisément les compétences en considérant les

\footnotetext{
${ }^{1}$ Ce sont nos traductions en français des référentiels génériques.
} 


\section{$\&$}

\section{REVUE HYBRIDE DE L'ÉDUCATION}

mêmes tâches, mais pour un domaine (discipline, faculté) ou un niveau d'étude (baccalauréat, maîtrise, doctorat) particulier.

Durant l'étape 3, on approfondit systématiquement chaque compétence spécifique en listant toutes ses sous-compétences. On décrit ensuite précisément celles-ci avec la méthode de Paquette (2002), qui permet un véritable approfondissement pédagogique, en esquissant les activités 1) d'apprentissage, puisqu'elle décrit les actions mentales des objectifs d'apprentissage, et 2) d'évaluation des compétences puisque la taxonomie des habiletés et connaissances établit des niveaux de difficulté. Continuons toujours le même exemple: les sous-compétences de la compétence spécifique "Choisir et Utiliser des bases de données bibliographiques scientifiques pour faire une recherche d'information thématique » sont :

1. Connaissance des bases de données bibliographiques ;

2. Choix d'une base de données bibliographiques ;

3. Création d'une requête thématique ;

4. Évaluation d'une requête thématique ;

5. Utilisation d'une base de données bibliographiques pour faire une requête thématique ;

6. Amélioration-Correction d'une requête thématique.

Dans le modèle de Paquette, la sous-compétence 1 peut être décrite avec plusieurs mobilisations d'habiletés sur des connaissances: a) Réception-Mémoriser x Principe (l'étudiant sait qu'une base de données bibliographiques scientifique est systématique), et b) ReproductionInstancier x Concept (l'étudiant peut préciser que Google n'est pas une base de données bibliographiques scientifique). De même, la souscompétence 3 peut être décrite avec: a) Réception-Mémoriser $x$ Procédure (l'étudiant connaît la notion de mots-clés), et b) CréationAnalyser x Procédure (l'étudiant sait créer une liste de mots-clés).

\section{Référentiel de compétences dans les situations du métier de l'étudiant·e}

Sur la base des éléments relatifs aux situations, l'équipe genevoise a adopté une approche complémentaire qui consiste à approfondir un référentiel de compétences numériques en le contextualisant par les situations (tableau 2). Nous partons ainsi d'un référentiel générique qui est adapté au contexte de l'institution universitaire, puis approfondi par des situations d'apprentissage. On utilise ici la méthode d'écriture des compétences de Bégin (2008). 


\section{REVUE HYBRIDE DE L’ÉDUCATION}

\section{Tableau 2 : Construction d'un référentiel contextualisé par les situations de travail}

\begin{tabular}{|c|c|}
\hline \multicolumn{2}{|c|}{ Étape 1 : Focalisation et alignement des compétences } \\
\hline $\begin{array}{l}\text { Actions concrètes effectuées } \\
\text { - prendre le référentiel générique } \\
\text { - adapter le référentiel générique }\end{array}$ & $\begin{array}{l}\text { Éléments définis dans le référentiel } \\
\text { - intitulés des compétences } \\
\text { génériques descriptions courtes des } \\
\text { - compétences génériques } \\
\text { - hiérarchie des compétences } \\
\text { génériques en } \\
\text { - groupes de compétences } \\
\text { génériques } \\
\text { niveaux de compétences } \\
\text { génériques } \\
\text { un exemple illustratif» } \\
\text { contextualisé : métier de sous- } \\
\text { compétences pour chaque } \\
\text { niveau de chaque compétence } \\
\text { générique }\end{array}$ \\
\hline \multicolumn{2}{|c|}{ Étape 2 : Contextualisation des compétences pour chaque situation } \\
\hline $\begin{array}{l}\text { Actions concrètes effectuées } \\
\text { - rendre le référentiel spécifique } \\
\text { - contextualiser avec les compétences } \\
\text { métiers } \\
\text { - contextualiser avec les situations }\end{array}$ & $\begin{array}{l}\text { Éléments définis dans le référentiel } \\
\text { - intitulés des situations pour les } \\
\text { compétences métiers } \\
\text { - descriptions courtes des situations } \\
\text { - listes des compétences } \\
\text { spécifiques mobilisées } \\
\end{array}$ \\
\hline \multicolumn{2}{|c|}{ Étape 3 : Approfondissement des compétences pour chaque situation } \\
\hline $\begin{array}{l}\text { Actions concrètes effectuées } \\
\text { - lister les sous-compétences de chaque } \\
\text { situation } \\
\text { - décrire précisément chaque sous- } \\
\text { compétence } \\
\text { - en conséquence, décrire précisément } \\
\text { chaque compétence }\end{array}$ & $\begin{array}{l}\text { Éléments définis dans le référentiel } \\
\text { - liste de compétences spécifiques } \\
\text { mobilisées pour chaque situation } \\
\text { - descriptions approfondies des } \\
\text { sous-compétences sous forme } \\
\text { de : } \\
\text { - situations de performance, } \\
\text { - production de connaissances } \\
\text { - exécution de tâches } \\
\text { d'apprentissage }\end{array}$ \\
\hline
\end{tabular}

À la fin de l'étape 1, on dispose d'un référentiel de compétences adapté de plusieurs référentiels génériques de base dont on a sélectionné des groupes de compétences. La sélection de ces référentiels d'origine se fait sur la base du métaréférentiel du Digital Quotient (DQ) (Coalition for Digital Intelligence, 2020) qui permet d'identifier DigComp comme 


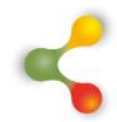

\section{REVUE HYBRIDE DE L'ÉDUCATION}

référentiel générique de base et de le compléter par des éléments d'autres référentiels, dont celui du JISC (JISC team, 2014). Le référentiel obtenu se décline en groupes de compétences, compétences génériques et niveaux de compétences. On désigne ici par compétences génériques des compétences purement numériques. Ainsi, le premier des quatre groupes, "Traitement de l'information et des médias", se scinde en trois compétences génériques: 1) Recherche d'informations/données ; 2) Filtrage et évaluation, et 3) Gestion, stockage et réutilisation. De plus, on distingue quatre niveaux de compétences : A) minimal, B) indépendant, C) expert, D) transmission de compétences.

Lors de l'étape 2, on introduit les compétences métiers de façon transversale aux compétences génériques. Ce sont des compétences numériques contextualisées selon les activités des étudiant·e.s dans leur métier d'apprenant·e.s. Dans notre cas, elles sont principalement extraites des profils d'apprenant (learner profile) (JISC team, 2014). Cette compétence métier est déclinée en situations d'apprentissage où sont mobilisées les composantes spécifiques de plusieurs compétences génériques. Puis chaque situation est associée aux compétences génériques mobilisées. On introduit, par exemple, la compétence métier " Je sais employer des outils numériques pour organiser, planifier et gérer l'apprentissage ", à laquelle correspondra la situation possible suivante ; "Dans le cadre d'un travail collaboratif pour un cours, je fais partie d'un groupe de cinq personnes. Nous allons faire des recherches individuelles, les partager avec les autres membres du groupe, réaliser une courte enquête puis rédiger collaborativement une synthèse. "

Enfin, lors de l'étape 3, chaque situation est décrite pour chaque niveau de chaque compétence générique activée sous forme de souscompétences, toujours selon le modèle proposé par Bégin (2008). Chaque situation est donc systématiquement approfondie et enrichie par les descriptions des sous-compétences génériques nécessaires à sa réalisation. Pour poursuivre avec le même exemple, une sous-compétence réalisable dans cette situation sera : "Lors de la recherche personnelle, je suis capable de sauvegarder dans un fichier les informations que je trouve pour pouvoir les retrouver plus tard. " Elle correspond au niveau A de la compétence 3 (recherche d'informations/données) du groupe de compétences « traitement de l'information et des médias ». Une autre souscompétence de la situation peut s'exprimer sous la forme : "Lors de la recherche personnelle, je suis capable de classer avec méthode les informations que je trouve en utilisant des fichiers et des dossiers afin de les retrouver facilement. » Elle correspond au niveau B de la compétence 3 (recherche d'information/données) du groupe de compétences « traitement de l'information et des médias". Si ces compétences ne sont pas exprimées en réutilisant explicitement la taxonomie de Bégin, on voit qu'elles peuvent s'y rattacher. 


\section{$\&$}

\section{REVUE HYBRIDE DE L'ÉDUCATION}

\section{Apports et prospectives}

Procédons à notre exploration des aspects de la médiation pédagogique aidés par le référentiel de compétences écrit et structuré comme décrit précédemment. En parlant d'un référentiel court, nous signifions qu'il est construit en suivant les étapes 1 et 2 . La construction d'un référentiel approfondi inclut l'étape 3. Dans les expériences décrites, nous explicitons le travail fait et la méthode suivie, les aides apportées par le référentiel et les aspects de la médiation pédagogique touchés. Nous ne détaillons pas les résultats obtenus, mais bien les activités de médiation que ces référentiels permettent de mettre en place.

Les expériences faites à Fribourg sont en relation avec la création d'un cours ; nous insistons donc plus sur la médiation entre enseignant.e.s et étudiant.e.s. Les expériences faites à Genève s'inscrivent dans une démarche institutionnelle; nous insistons sur une médiation plus large entre étudiant·e.s, enseignant·e.s et institution.

\section{Enquête globale sur les compétences-objectifs}

Une enquête menée à l'Université de Fribourg en décembre 2019 a permis de demander aux étudiant.e.s d'évaluer leur niveau de maîtrise des compétences numériques et l'utilité de celles-ci pour leur cursus. Le référentiel court permet une telle analyse globale parce qu'il repose sur les groupes de compétences spécifiques qui permettent d'établir un questionnaire cohérent et compréhensible du point de vue de la médiation pédagogique :

- aspect conceptualisation : la focalisation établit la structure du questionnaire sur la base des groupes de compétences en facilitant le regroupement/séparation des questions posées en lien avec les objectifs principaux du cours ;

- aspect langage: la contextualisation rend les intitulés des compétences-objectifs compréhensibles, car on parle d'activités que les étudiant.e.s font.

\section{Profil de compétences des activités des étudiant.e.s}

En décrivant les compétences et sous-compétences spécifiques selon la méthode de Paquette, nous listons toutes les actions mentales impliquées par un·e étudiant·e effectuant une activité spécifique. Par exemple, pour l'activité spécifique « Choisir et utiliser une base de données bibliographiques scientifiques pour une recherche d'information thématique ", on définit quarante actions réparties dans six souscompétences ("Connaissance des bases de données bibliographiques », "Création d'une requête thématique », etc.). 


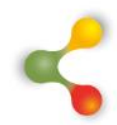

\section{REVUE HYBRIDE DE L'ÉDUCATION}

Ces sous-compétences organisent la liste des actions identifiées pour l'activité spécifique complète : c'est la hiérarchie systématique des compétences et sous-compétences (tableau 1). Dans cette tâche de description, la collaboration de plusieurs enseignant.e.s pour lister ces actions permet de trouver le nombre des actions à prendre en compte et, surtout, d'établir des profils taxonomiques de compétences pour l'activité analysée (niveaux d'habiletés et types de connaissances différents).

Cette description et cette organisation du référentiel approfondi, basées sur la méthode de Paquette, permettent plusieurs aides à la médiation pédagogique :

- Les sous-compétences distinguent des sous-activités préfigurant des activités d'apprentissage à proposer aux étudiant $\cdot e \cdot s$, traçant ainsi une organisation de l'intervention éducative (médiation psychopédagogique) et du travail de l'étudiant·e (médiation cognitive).

- La définition des niveaux d'habiletés et des types de connaissances mobilisées pour les différentes sous-compétences facilite un travail métacognitif de conceptualisation pour la compétence spécifique entière, selon les niveaux d'habiletés majoritaires ou connaissances mobilisées.

\section{Réalisation d'un test d'autopositionnement}

Un outil d'autopositionnement est développé pour les étudiant $\cdot e \cdot s$ de l'Université de Genève à partir du référentiel approfondi. II doit être complet par rapport aux compétences génériques du référentiel et le plus concis possible afin que l'apprenant·e le réalise en entier. On questionne l'apprenant $e$ à partir des situations parce que le contexte auquel elles sont rattachées permet plus facilement à l'étudiant·e d'évaluer sa maîtrise de la compétence. Nous définissons un ensemble minimal de situations qui couvrent toutes les compétences génériques du référentiel et où chaque compétence du référentiel est activée au moins deux fois. On peut présenter ainsi la même compétence générique au moins deux fois et s'appuyer sur les réponses de l'apprenant $e$ aux deux situations différentes pour décider son niveau de maîtrise.

Au final, le test consiste à présenter successivement à l'étudiant·e plusieurs situations contextualisées de son métier d'apprenant·e. Pour chacune, on demande si elle ou il se sent capable de la réaliser (pas du tout, plutôt pas, assez, tout à fait), avec une sous-compétence d'apprentissage correspondant au niveau d'une compétence générique. En fonction de la réponse donnée, des sous-compétences d'un niveau inférieur ou supérieur sont présentées. Le niveau de l'étudiant·e pour la compétence générique est déterminé en combinant les réponses aux deux niveaux. 


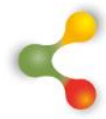

\section{REVUE HYBRIDE DE L'ÉDUCATION}

\section{Dialogue sur un changement de pratique numérique}

La situation imposée par la pandémie de COVID-19 a changé les conditions d'apprentissage de façon brutale. À l'Université de Genève, les cours sont passés en quelques jours d'un mode présentiel ou hybride à un mode complètement distanciel. Identifier les conséquences d'un tel changement sur l'évolution des compétences numériques est important. Là encore, l'approche du référentiel approfondi basé sur les situations d'apprentissage permet d'interroger les étudiant·es.s en partant des situations d'apprentissage et d'utiliser une décomposition sous forme de compétences numériques génériques. Pour chaque situation pertinente du référentiel, les descriptions des sous-compétences spécifiques associées sont présentées aux étudiant·e.s en leur demandant si chacune est toujours activée pour la réalisation de la situation. Des sous-compétences supplémentaires précises, basées par exemple sur les observations des enseignant.e.s ou des nouveaux outils numériques mis en place, peuvent aussi être présentées. Enfin, les étudiant.e.s peuvent également indiquer librement s'ils ont activé d'autres sous-compétences et les formuler eux.elles-mêmes (analyse parfois complexe).

\section{Conclusions et perspectives}

Nous avons passé en revue les apports des référentiels de compétences dans les processus de médiation pédagogique. Les caractéristiques particulières des compétences numériques nous ont amenés à considérer que, pour permettre d'opérationnaliser les missions de médiation pédagogique d'un référentiel générique, il est nécessaire de l'approfondir en le contextualisant. Nous avons ainsi présenté deux approches de contextualisation en trois étapes, une utilisant le modèle des activités de Paquette (2002) et une autre, le modèle des situations de Bégin (2008). Nous avons conçu ainsi deux référentiels et avons décrit ensuite différentes expériences de leurs utilisations pour une médiation pédagogique.

Nous concluons que les deux référentiels sont complémentaires, car ils ouvrent des portes d'entrée différentes (par les activités ou par les situations) pour définir des ressources sur les compétences numériques. Nos expériences révèlent l'apport des référentiels dans la médiation pédagogique, en particulier pour définir un langage commun permettant la communication et la conceptualisation. Toutefois, ces démarches de contextualisation approfondie démultiplient les efforts pour libeller les compétences selon des structures hiérarchiques permettant l'alignement et la différenciation entre les groupes de compétences. La transversalité des compétences numériques selon les disciplines peut également nécessiter de les contextualiser au moins partiellement. 


\section{8}

\section{REVUE HYBRIDE DE L'ÉDUCATION}

Nous désirons continuer à étudier la complémentarité des deux référentiels et voir dans quelle mesure elle pourrait s'enrichir avec, peutêtre, un référentiel à plusieurs portes d'entrée. Deux directions de travaux futurs sont envisagées :

- pour l'utilisation du référentiel, comme squelette de tests d'autopositionnement et de recommandations de ressources ainsi que pour développer de nouvelles activités de cours ;

- pour le développement du référentiel : pour des enseignant·e.s ou chercheur.e.s et pour spécialiser les référentiels pour des disciplines particulières. 


\section{REVUE HYBRIDE DE L'ÉDUCATION}

\section{Références}

Bégin, C. (2008). Les stratégies d'apprentissage : Un cadre de référence simplifié. Revue des sciences de l'éducation, 34(1), 47-67. https://doi.org/10.7202/018989ar

Carignan, L., Fourdrignier, M. et Lindsay, J. (2014). Pratiques réflexives et référentiels de compétences dans les formations sociales. Presses de I'Université du Québec.

Carretero, S., Vuorikari, R. et Punie, Y. (2017). DigComp 2.1: The Digital Competence Framework for Citizens with eight proficiency levels and examples of use (EUR 28558 EN). European Commission. http://publications.jrc.ec.europa.eu/repository/bitstream/JRC106281 /web-digcomp2.1pdf_(online).pdf

Chini, D. (2001). Médiation(s): Quelques remarques sur un terme pluriel. ASp. La revue du GERAS, 31-33, 133-142. https://doi.org/10.4000/asp.1901

Coalition for Digital Intelligence. (2020). DQ (Digital Intelligence) Framework. https://www.dqinstitute.org/dq-framework/

Gillet, P. (1991). Construire la formation. ESF.

Gouvernement de l'Ontario. (2016). Phase 1-Définir les compétences du 21e siècle pour l'Ontario: Document de réflexion. Ministère de l'Éducation de l'Ontario. https://pedagogienumeriqueenaction.cforp.ca/wpcontent/uploads/2016/02/Ontario-21stcentury- competenciesfoundation-FINAL-FR AODA EDUGAINS Feb-19 16.pdf

JISC team. (2014). Developing digital literacies. Full guide http://web.archive.org/web/20141011143516/http://www.jiscinfonet. ac.uk/infokits/digital-literacies/

Jones, C. (2013). The digital university. A concept in need of definition. Dans R. Goodfellow, L. Goodfellow et R. Mary (dir.), Literacy in the digital university: Critical Perspectives on learning, scholarship, and technology (p. 162-172). Routledge.

Le Boterf, G. (1999). De la compétence à la navigation professionnelle. Éditions d'Organisation.

Lenoir, Y. (2009). L'intervention éducative, un construit théorique pour analyser les pratiques d'enseignement. Nouveaux cahiers de la recherche en éducation, 12(1), 9-29.

Littlejohn, A., Beetham, H. et McGill, L. (2013). Digital literacies as situated knowledge practices. Academics' influence on learners' behaviours. Dans R. Goodfellow et M. R. Lea (dir.), Literacy in the digital university (p. 126-136). Routledge. 


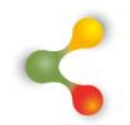

\section{REVUE HYBRIDE DE L'ÉDUCATION}

Lowe, M. S., Booth, C., Stone, S. et Tagge, N. (2015). Impacting Information Literacy Learning in First-Year Seminars: A RubricBased Evaluation. Portal-Libraries and the Academy, 15(3), 489-512. https://doi.org/10.1353/pla.2015.0030

Mayen, P., Métral, J.-F. et Tourmen, C. (2010). Les situations de travail. Références pour les référentiels. Recherche et formation, 64, 31-46. https://doi.org/10.4000/rechercheformation.191

Gouvernement du Québec. (2019). Cadre de référence de la compétence numérique. Ministère de l'Éducation et de l'Enseignement supérieur du Québec.

http://www.education.gouv.qc.ca/fileadmin/site web/documents/min istere/Cadre-referencecompetence- num.pdf

Myara, N. (2017). Le plan d'intervention: Un processus et des ententes. Éditions JFD.

Myara, N. (2018a). La médiation pédagogique! Quoi ? Qui ? Quand et comment? (1 ̀̀re partie). Vivre le primaire, printemps 2018, 68-71.

Myara, N. (2018b). La médiation pédagogique! Quoi ? Qui ? Quand et comment? (2 $2^{\mathrm{e}}$ partie). Vivre le primaire, été 2018, 80-83.

Paquette, G. (2002). Modélisation des connaissances et des compétences. Un langage graphique pour concevoir et apprendre. Presses de l'Université du Québec.

Peraya, D., Charlier, B. et Deschryver, N. (2014). Une première approche de l'hybridation Étudier les dispositifs hybrides de formation. Pourquoi ? Comment ?. Éducation et Formation, e-301, 15-34.

Piaget, J. (1971). La causalité selon E. Meyerson. Dans M. Budge, F. Halbwachs, T.S. Kuhn et L. Rosenfeld (dir.), Les théories de la causalité (p. 151-208). Presses universitaires de France.

Platteaux, H., Salietti, E. et Molteni, L. (2019). The design of a rubric for defining and assessing digital education skills of higher education students. European Journal of Open, Best of EDEN2018 Special Issue, 140-151.

Postiaux, N., Bouillard, P. et Romainville, M. (2010). Référentiels de compétences à l'université. Usages, rôles et limites. Recherche et formation, 64, 15-30.

Rabardel, P. et Samurcay, R. (2001). From artifact to instrument-mediated learning. Dans Center for Activity Theory and Developmental Work Reseach (resp.), Symposium on New challenges to research on Learning [symposium]. New Challenges to Research on Learning, Helsinki, Finlande. file:///C:/Users/cathe/Downloads/2001-RabardelSamur\%C3\%A7ayChallenges\%20Research\%20on\%20Learning.pdf 


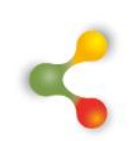 \\ REVUE HYBRIDE DE L'ÉDUCATION}

Rey, B. (2006). Les compétences professionnelles et le curriculum: Des réalités conciliables? Dans Y. Lenoir et M.-H. Bouillier-Oudot, Savoirs professionnels et curriculum de formation (p. 83-107). Presses Université Laval.

Rogiers, X. et De Ketele, J.-M. (2001). Une pédagogie de l'intégration. Compétences et intégration des acquis dans l'enseignement. De Boeck Université.

Rusman, E. et Dirkx, K. (2017). Developing Rubrics to Assess Complex (Generic) Skills in the Classroom : How to Distinguish Skills' Mastery Levels?. Practical Assessment, Research \& Evaluation, 22(12). http://pareonline.net/getvn.asp? $\mathrm{v}=22 \& \mathrm{n}=12$

Scallon, G. (2004). L'évaluation des apprentissages dans une approche par compétences. De Boeck.

Sorgo, A., Bartol, T., Dolnicar, D. et Podgornik, B. B. (2017). Attributes of digital natives as predictors of information literacy in higher education. British Journal of Educational Technology, 48(3), 749-767. https://doi.org/10.1111/bjet.12451

Tardif. (2006). L'évaluation des compétences: Documenter le parcours de développement. Chenelière Éducation.

Tardif, M. et Lessard, C. (1999). Le travail enseignant au quotidien. Contribution à l'étude du travail dans les métiers et les professions d'interactions humaines. Les Presses de l'Université Laval.

Van Laar, E., Van Deursen, A. J. A. M., Van Dijk, J. A. G. M., et De Haan, J. (2017). The relation between 21 st-century skills and digital skills: A systematic literature review. Computers in Human Behavior, 72, 577-588. https://doi.org/10.1016/j.chb.2017.03.010

Vandeweerd, J.-M., Cambier, C., Romainville, M., Perrenoud, P., Desbrosse, F., Dugdale, A. et Gustin, P. (2014). Competency Frameworks: Which Format for Which Target?. Journal of Veterinary Medical Education, 41(1), 27-36. https://doi.org/10.3138/jvme.0413062R1

Varinot, A. (2012). La médiation pédagogique au service de la métacognition. [Mémoire de maîtrise, Université d'Orléans]. https://dumas.ccsd.cnrs.fr/file/index/docid/760968/filename/Memoir e M2 MEEFA Anthony Varinot.pdf

Voogt, J. et Roblin, N. P. (2012). A comparative analysis of international frameworks for $21^{\text {st }}$ century competences: Implications for national curriculum policies. Journal of Curriculum Studies, 44(3), 299-321. https://doi.org/10.1080/00220272.2012.668938

Vygotski, L. S. (1985). Pensée et langage (traduit par F. Sève). MessidorÉditions sociales. 